\title{
Polycystic Ovary Syndrome and the Role of Metformin in Ovulation Induction
}

\author{
Fabiana Fraga1, Gabriel Azeredo César Salgado Romeiro², \\ Larissa Bianca Paiva Cunha de Sá1, Alberto Krayyem Arbex ${ }^{1,3,4}$ \\ ${ }^{1}$ Endocrinology Division, IPEMED Medical School, São Paulo, Brazil \\ ${ }^{2}$ Internal Medicine Division, Itajubá, Brazil \\ ${ }^{3}$ Diabetology Department, Malteser Krankenhaus St. Franziskus Hospital, Flensburg, Germany \\ ${ }^{4}$ Harvard T. H. Chan School of Public Health, Boston, MA, USA \\ Email: albertoarbex@gmail.com
}

How to cite this paper: Fraga, F., Romeiro, G.A.C.S., de Sá, L.B.P.C. and Arbex, A.K. (2018) Polycystic Ovary Syndrome and the Role of Metformin in Ovulation Induction. Health, 10, 565-576.

https://doi.org/10.4236/health.2018.105045

Received: March 21, 2018

Accepted: May 21, 2018

Published: May 24, 2018

Copyright (c) 2018 by authors and Scientific Research Publishing Inc. This work is licensed under the Creative Commons Attribution International License (CC BY 4.0).

http://creativecommons.org/licenses/by/4.0/

Open Access

\begin{abstract}
The Polycystic Ovary Syndrome (PCOS) is frequently associated with comorbidities such as obesity, reduced glucose tolerance, hypertension, macrovascular disease and dyslipidemia. The Metabolic syndrome occurs in $30 \%$ of women with PCOS. Metformin has increasingly been used in this therapy due to its effects in reducing insulin resistance. Treatment of PCOS aims to reduce the symptoms of hyperandrogenism, regularize the menstrual cycle, reduce metabolic abnormalities, and lower the risk of type 2 diabetes mellitus and of cardiovascular disease. Additionally it is important to prevent hyperplasia and endometrial cancer, and to offer contraception to those who do not wish pregnancy, and to help to induce ovulation to those who do. The effectiveness of metformin in this treatment is assessed in the light of the current best evidence.
\end{abstract}

\section{Keywords}

Polycystic Ovary Syndrome, Metformin, Oral Contraceptives, Fertility, Anovulation

\section{Introduction Methodology and Goals}

The polycystic ovary syndrome (PCOS) is an endocrinopathy commonly occurring in reproductive age, affecting up to one in five women, depending on the population studied and the diagnostic criteria used, and it is the single most common cause for infertility in women [1] [2].

It is estimated that, worldwide, 105 million women aged between 15 and 49 years are affected by PCOS [3]. However, there are few epidemiologic studies on 
PCOS, especially studies based on population [4].

It was originally described by Stein and Leventhal as an association of amenorrhea and polycystic ovaries. Five of the seven original cases displayed hirsutism or acne, and four displayed obesity [5]. Several diagnostic criteria and consensuses have been created over time. Of those, the one that is currently most widely accepted is the Rotterdam consensus (2004), confirmed by the National Institute of Health (NIH)-USA (2012). According to the consensus, at least two of three established criteria are necessary: 1) Oligo- and/or anovulation; 2) Clinical and/or biochemical hyperandrogenism; 3) Polycystic ovaries; as well as the exclusion of other etiologies with similar clinical manifestation, such as: nonclassic (late-onset) congenital adrenal hyperplasia, androgen-secreting tumors, thyroid dysfunction, hyperprolactinemia, Cushing's syndrome, ovarian insufficiency, hypothalamic amenorrhea, and the use of exogenous androgens [2] [6].

Many women with PCOS display characteristics such as obesity, reduced glucose tolerance, hypertension, macrovascular disease, and dyslipidemia, which are common in metabolic syndrome [7] [8] [9]. The prevalence of metabolic syndrome in women with PCOS is approximately $30 \%$, according to several studies [2].

Though the pathophysiology of metabolic syndrome is not clearly defined, recent evidence has accrued underscoring the role of insulin resistance as the basis of metabolic syndrome, as is described in the pathophysiology of PCOS [7].

The goal of this paper is to discuss the use of metformin in PCOS, its advantages, side-effects, and benefits in comparison to the standard treatment with oral contraceptives.

The methodology used was a review of present medical literature in search of the best available evidence for such treatment. Articles published in the past 10 to 15 years were reviewed in order to develop understanding of current tendencies of therapeutics and of the still-unclear points of treatment options available. The search was performed in the databases: PUBMED, EMBASE, BIREME, UPTODATE with "polycystic ovarian syndrome, metformin, oral contraceptive, fertility and anovulation".

\section{Metformin and Metabolic Syndrome}

Women with metabolic syndrome have been demonstrated to display an increase in proinflammatory cytokines produced by the adipose tissue in substantial quantity, such as tumor necrosis factor alpha and interleukin 6, which appear to compromise the effect of insulin on the cellular uptake of glucose and to induce endothelial inflammatory response [7].

$\mathrm{C}$ reactive protein, a sensitive acute-phase inflammatory marker, has a positive correlation with insulin resistance, compensatory hyperinsulinemia, and some manifestations of metabolic syndrome, as well as an independent association with the risk of coronary artery disease. Thus, associated with visceral fat, insulin resistance appears to result in endothelial dysfunction, vascular damage, oxidative stress at the endothelial level, and subsequent formation of atheroma plaque [7]. 


\section{Metformin and the Polycystic Ovary Syndrome}

The use of metformin in the treatment of PCOS has been under debate in the past decade. Most earlier studies indicated that metformin improved hyperinsulinemia and hyperandrogenemia and restored ovulatory function, and that its use, either on its own or combined with clomiphene citrate, could increase ovulation and pregnancy rates in women with PCOS [6]. Furthermore, nonrandomized prospective studies suggested that metformin may reduce first-trimester spontaneous abortions in women with PCOS. However, more recent large clinical randomized trials have shown controversial results, with either a beneficial effect or no effect of metformin on fertility in PCOS [6].

The Endocrine Society have conducted a systematic analysis and published a consensus on cardiovascular risk assessment and prevention of cardiovascular disease in women with PCOS. In addition to an increase in triglycerides and a decrease in high-density lipoproteins (HDL), women with PCOS have increased low-density lipoprotein (LDL) and non-cholesterol HDL levels, regardless of BMI. Women with PCOS should have their BMI and blood pressure measured at each clinic visit and, once PCOS has been diagnosed, a full lipid profile should be included [6].

Even though hypertension has been an inconsistent finding, women with PCOS appear to be at a later risk. Although in many studies both systolic and diastolic blood pressures are normal, in other studies mean arterial pressures and ambulatory systolic pressures are elevated in women with PCOS in comparison with the controls. Anatomic evidence of early-onset coronary artery disease and of other vascular diseases in PCOS has been documented through varied techniques. Increase in carotid artery intima-media thickness, an independent predictor of cerebrovascular accident and myocardial infarction, has been observed in PCOS compared with control women of similar age. Calcification of the coronary artery, another marker of atherosclerosis, is more common in women with PCOS than in controls, even after adjustment for the effects of age and BMI [6].

\section{Therapeutic Approach}

Treatment of PCOS aims to reduce the symptoms of hyperandrogenism, regularize the menstrual cycle, reduce metabolic abnormalities, lower the risk of type 2 diabetes mellitus and of cardiovascular disease, prevent hyperplasia and endometrial cancer, and offer contraception to those who do not wish pregnancy and ovulation induction to those who do [2].

The strategy of lifestyle modification comprising diet associated with regular physical exercise is essential, particularly for women with central obesity [2] [10] [11].

As a consequence of lifestyle modification, a reduction of $7 \%-10 \%$ in body weight should occur within 6 to 12 months, even though the goal is a reduction of $10 \%-20 \%$. With that, regularization of menses and spontaneous ovulation 
are achieved, as well as improvement of insulin resistance, dyslipidemia, DM2, depression, and quality of life [2] [12] [13].

The first-line pharmacological treatment is the use of hormonal contraceptive methods, specifically Combined Oral Hormonal Contraceptives (COCs), in women with PCOS who do not wish pregnancy, aiming to regularize menses and improve hirsutism. COCs may also be used as an isolated drug in cases of mild hirsutism [2] [14].

\section{Available Combined Oral Hormonal Contraceptives (COCs)}

Combined Oral Hormonal Contraceptives composed of progestins suppress LH and consequently reduce the production of ovarian androgens. The estrogenic fraction of the pill stimulates hepatic production of SHBG, increasing serum androgen binding and reducing the concentration of circulating unbound androgens and their bioactivity. COCs also have an antiproliferative effect on the endometrium [2] [15].

Other hormonal methods may also be used similarly to combined hormonal contraceptives (vaginal ring, patch, and monthly injectable), or composed only of progestins (oral, trimonthly injectable, subdermal implant, and intrauterine device) [2] [14].

In hirsute women, COCs containing neutral progestins or progestins with anti-androgenic activity are the method of choice for treatment. The progestins that have the most anti-androgenic activity are: cyproterone acetate, followed by the compounds of chlormadinone acetate, dienogest, drospirenone, nomegestrol; in addition to those with weak androgenic activity: desogestrel, gestodene, and norgestimate; and those with androgenic effects, such as levonorgestrel and norethisterone acetate [2].

Daily doses of ethinylestradiol that are considered low $(20-35 \mu \mathrm{g})$ are sufficient to suppress follicular activity and reduce the symptoms of hyperandrogenism. Therefore, improvement in hirsutism and acne are observed within at least six months of use of COCs, given that the growth cycle of hair varies greatly in different areas of the body [2].

Among the antiandrogens, cyproterone acetate can be used in orally-administered 25 to $50-\mathrm{mg}$ doses, from the 5 th to the 14 th day; or spironolactone in orally-administered 25 to $100-\mathrm{mg} /$ day doses, both associated with COCs. Effective contraception is necessary when using antiandrogens, since they can cause feminization of male fetuses [2] [14].

The benefits of hormonal contraceptives outweigh the risks for the vast majority of patients with PCOS. However, potentially adverse metabolic and cardiovascular effects are a concern, seeing that the treatment lasts several years. Therefore, the choice of hormonal methods should be individualized depending on each patient's characteristics and on the presence of contraceptive risk factors, and a detailed screening of possible contraindications should be the basis 
for the decision [2].

In obese women with PCOS, preference should be given to natural COCs, such as 17-beta estradiol and estradiol valerate, which reduce metabolic effects when compared to COCs containing ethinylestradiol [2].

\section{The Role of Metformin in the Treatment of PCOS}

\section{1) Metformin for control of menstrual cycles}

Metformin is a potential alternative for regularizing menstrual cycles because it restores ovulatory menses in approximately 30 to 50 per cent of women with PCOS [16] [17]. Its capacity to provide endometrial protection is far less well-established and, consequently considered second-line therapy [18] [19].

When metformin is used, follow-up is suggested to confirm whether ovulatory cycles have been established. This can be done by measuring luteal phase serum progesterone or by performing a transvaginal ultrasound [14].

Interest in the use of metformin increased when the role of insulin resistance in the pathophysiology of the disease was noticed. Metformin is typically the first-line treatment for patients with type 2 diabetes; its use in pre-diabetes or PCOS is not approved according to the prescribing information, even though it is often prescribed for such conditions [20].

Early trials in women with PCOS later showed benefits in weight loss, reduction of androgens, and restoration of menses in approximately $50 \%$ of women with oligomenorrhea. Early data also suggested the efficacy of metformin for ovulation induction in women with PCOS. As a consequence, metformin has been used off-label for many of such indications [16] [17].

\section{2) Mechanism of action and dosage}

Metformin is an antihyperglycemic biguanide approved for management of type 2 diabetes mellitus when hyperglycemia cannot be controlled with lifestyle modification, i.e., diet and exercise. Contrary to sulfonylureas, it does not produce hypoglycemia in normal individuals or in patients with type 2 diabetes [20].

The main effect of metformin is to reduce the production of hepatic glucose, therefore reducing the need of insulin secretion; it also reduces intestinal glucose absorption. Metformin also has an antilipolytic effect that reduces the concentration of free fatty acids, thus lowering gluconeogenesis [18] [19].

Metformin is available in 500-, 850-, and 1000-mg tablets. The target dose is between $1500 \mathrm{mg}$ and $2000 \mathrm{mg}$ per day. Clinically significant responses are not regularly observed at doses smaller than $1000 \mathrm{mg}$ per day. Many clinicians start treatment with $500 \mathrm{mg}$ taken with a meal in order to reduce gastrointestinal side effects. If tolerated, the dose may be increased to $500 \mathrm{mg}$ at lunch and dinner, and then to $500 \mathrm{mg}$ at breakfast, lunch, and dinner. There should be one to two weeks between dosage increases [20].

The most common side effects are gastrointestinal, such as diarrhea, nausea or vomiting, flatulence, indigestion, and abdominal discomfort. Such symptoms are 
generally mild, transitory, and reversible upon reduction of dosage or discontinuation of the medication [20].

\section{3) Side effects}

For women with PCOS who take metformin, we suggest annual monitoring of B12 deficiency with full blood count and B12 concentration. In addition, they should be certain to meet the recommended B12 dietary provisions $(2.6 \mathrm{mcg}$ for adults). It is worth noting that metformin reduces intestinal absorption of vitamin B12 in up to 30 percent of patients and it reduces serum concentration of vitamin B12 by 5 to 10 percent, but it rarely causes megaloblastic anemia [21].

Metformin should not be prescribed to women with conditions that increase the risk of lactic acidosis, such as renal insufficiency, congestive cardiac failure, or sepsis, even though lactic acidosis is considered an extremely rare complication in healthy individuals [22] [23].

\section{4) Efficacy}

Data indicate that metformin will restore ovulatory menses in approximately $50 \%$ of women with PCOS, even though some studies report ovulatory rates from $23 \%$ to $90 \%$ [24] [25]. Evidence of the efficacy of metformin includes a trial involving 23 women with PCOS who were randomly designated to receive either metformin (500 mg three times a day) or placebo for six months [24]. Approximately half of the women treated with metformin achieved normalization of the menstrual function, confirmed by intermenstrual interval and by monitoring of the luteal phase serum progesterone. In a meta-analysis of 13 trials, women treated with metformin had four times as much probability of ovulation as the women treated with placebo [23] [26]. A similar fourfold increase was observed for the combination of metformin and clomiphene when compared with clomiphene alone [20].

According to Palomba S., et al., a six-month course of treatment with metformin $1700 \mathrm{mg} /$ day and clomiphene citrate, administered in an escalation protocol, are two first-line approaches to improve fertility in women with PCOS [27].

Some women do not ovulate during the first six months of treatment; we therefore recommend treatment with cyclical progestin in this interval. Although some women may restore the menstrual cycle with metformin, unless the cycles are regular and ovulatory, it cannot be assumed that the patient has reliable endometrial protection [20].

For those women who have irregular and anovulatory menstrual cycles, cyclical progestins or COCs are necessary. Normally, micronized progesterone $(200 \mathrm{mg} /$ day for 14 days each two months) or medroxyprogesterone acetate $(10 \mathrm{mg} /$ day for 12 to 14 days each one to two months) are prescribed. Women who do not achieve regular ovulatory menses should be treated with cyclical progestin therapy in order to reduce the risk of endometrial hyperplasia until regular menstruation is established [20].

After initiating therapy with metformin, it may take up to six months for the ovulatory cycles to occur. During the initial phase of oligomenorrhea, it may be 
difficult to determine whether or not ovulation has occurred [20].

\section{5) Contraceptives vs. Metformin in PCOS}

There are concerns that estrogen-progestin contraceptives worsen insulin resistance in women with PCOS and that therefore, metformin should be the treatment of choice. However, while COCs are less beneficial than metformin regarding insulin sensitivity, they are more effective in suppressing androgens and in controlling menstrual cycles. Moreover, the data available have not shown any evidence of adverse metabolic risk [28]. In a meta-analysis of four trials involving 104 women with PCOS, metformin was less effective than COCs in improving menstrual patterns and in reducing total testosterone serum concentration, but more efficient in reducing fasting insulin levels and in not increasing fasting triglyceride concentration [29].

In women with PCOS, administration of metformin before or during cycles of in vitro fertilization (IVF) does not appear to improve pregnancy or live birth rates [30] [31]. However, administration of metformin before or during cycles of IVF does appear to reduce the risk ovarian hyperstimulation syndrome (OHSS) [30] [32].

Although therapy with metformin is a potential option to reduce the risk of OHSS with IVF, there seem to be more effective strategies, such as gonadotropin-releasing hormone (GnRH) antagonists [33].

Addition of metformin in this configuration does not appear to reduce the risk of multiple gestation [34].

A meta-analysis of 13 trials reported that metformin is associated with a reduction of blood pressure, low-density lipoprotein (LDL), and fasting insulin levels, when compared with placebo [35].

At least $50 \%$ of women with PCOS are obese. For obese women with PCOS who need to lose weight, we suggest other weight loss medication or bariatric surgery rather than metformin. Nevertheless, some benefits are seen when metformin is combined with calorie restriction [20].

In one study, metformin ( $850 \mathrm{mg}$ twice a day) plus a low-calorie diet (1200 to 1400 kcal per day) was superior to a low-calorie diet alone, facilitating weight loss in obese women with and without PCOS [35]. Other researchers have reported similar results [36] [37] [38]. One trial reported that a higher dose of metformin $(2550 \mathrm{mg} /$ day) was more effective for weight loss than the lowest dose of metformin (1500 mg/day) in obese women with PCOS. The dose-dependent effect was observed in women with a BMI between 30 and 37 $\mathrm{kg} / \mathrm{m}^{2}$, but not in those whose BMI was higher than $37 \mathrm{~kg} / \mathrm{m}^{2}$ [39].

Gestational diabetes is considered a complication in $5 \%$ of pregnancies and it is associated with complications during pregnancy and long-term risk of diabetes both for mothers and offspring. Lifestyle modification intervention and, in case of persistent hyperglycemia, treatment with insulin have shown improvement in perinatal results. Women who initiate insulin therapy require education for safe administration of insulin. The use of insulin is also associated with hypoglycemia and weight gain. Therefore, the use of safe and effective oral 
agents may offer advantages over insulin [40].

Oral metformin is an option for women with gestational diabetes mellitus. It improves sensitivity to insulin and is not associated with weight gain or hypoglycemia. The reported results of its use during pregnancy have been favorable, except for a small retrospective cohort study that showed increased perinatal loss and pre-eclampsia rates compared to the treatment with insulin. According to the study by Janet A. R., et al., 2008, perinatal results were similar for both treatments (insulin versus metformin); however, the use of metformin was more acceptable to the women [40].

Given that PCOS is a chronic disorder affecting $5 \%$ to $10 \%$ of women of reproductive age, during the last decade, the use of metformin in such cases has been widely debated [41].

Most studies indicate that metformin improves hyperinsulinemia and hyperandrogenemia, as well as it restores ovulatory function; and that its use alone or combined with clomiphene citrate might increase ovulation and pregnancy rates in women with PCOS. Furthermore, a nonrandomized prospective study has suggested that metformin may reduce the risk of spontaneous abortion in the first trimester for women with PCOS [41].

The use of metformin alone or combined with other treatments for infertility, however, have improved pregnancy and live birth rates in women with PCOS; in addition, the most beneficial effects have been observed in obese women. This is an important result because the birth of a healthy child is the only meaningful result for a couple with infertility [41].

\section{Conclusions}

In order to minimize both short- and long-term repercussions for these patients, early detection and treatment of cardiovascular risk factors in patients with PCOS allows multidisciplinary interventions to be performed aiming to improve these women's quality of life.

PCOS treatment is symptomatic and driven by clinical manifestations, patients' wish for contraception or pregnancy, and the presence of associated metabolic disorders. Patients with PCOS should be informed that this is a chronic disease with long-term treatment and follow-up.

Lifestyle modifications, including diet associated with regular physical exercise, are considered the essential conduct, particularly for women with central obesity.

\section{References}

[1] Rosenfield, R.L. and Ehrmann, D.A. (2016) The Pathogenesis of Polycystic Ovary Syndrome (PCOS): The Hypothesis of PCOS as Functional Ovarian Hyperandrogenism Revisited. Endocrine Reviews, 2016, 367.

https://doi.org/10.1210/er.2015-1104

[2] Pontes, A. and Filho, B.S.A. (2016) Polycystic Ovarian Syndrome: Diagnosis, Treatment and Repercussions throughout Life. UNESP, Botucatu. 
[3] Azziz, R., Carmina, E., Dewailly, D., Diamanti-Kandarakis, E., Escobar-Morreale, H.F., Futterweit, W., Janssen, O.E., Legro, R.S., Norman, R.J., Taylor, A.E. and Witchel, S.F. and Androgen Excess Society (2006) Positions Statement: Criteria for Defining Polycystic Ovary Syndrome as a Predominantly Hyperandrogenic Syndrome: An Androgen Excess Society Guideline. The Journal of Clinical Endocrinology \& Metabolism, 91, 4237-4245. https://doi.org/10.1210/jc.2006-0178

[4] Ning, N., Balen, A., Brezina, P.R., Leong, M., Shoham, Z., Wallach, E.E. and Zhao, Y. (2013) How to Recognize PCOS: Results of a Web-Based Survey at IVF-Worldwide.com. Reproductive Biomedicine, 26, 500-505. https://doi.org/10.1016/j.rbmo.2013.01.009

[5] Sam, S., Legro, R.S., Essah, P.A., et al. (2006) Evidence for Metabolic and Reproductive Phenotypes in Mothers of Women with Polycystic Ovary Syndrome. Proceedings of the National Academy of Sciences of the United States, 103, 7030. https://doi.org/10.1073/pnas.0602025103

[6] Legro, R.S., Arslanian, S.A., Ehrmann, D.A., et al. (2013) Diagnosis and Treatment of Polycystic Ovary Syndrome: An Endocrine Society Clinical Practice Guideline. The Journal of Clinical Endocrinology \& Metabolism, 98, 4565. https://doi.org/10.1210/jc.2013-2350

[7] Costa, L.O.B.F., Viana, A.O.R. and Oliveira, M. (2007) Prevalence of Metabolic Syndrome in Patients with Polycystic Ovary Syndrome. Revista Brasileira de Ginecologia e Obstetrícia 29, 10-17.

[8] Twig, G., Yaniv, G., Levine, H., et al. (2016) Body-Mass Index in 2.3 Million Adolescents and Cardiovascular Death in Adulthood. The New England Journal of Medicine, 374, 2430. https://doi.org/10.1056/NEJMoa1503840

[9] Fauser, B.C. and Bouchard, P. (2011) Uncertainty Remains in Women with PCOS Regarding the Increased Incidence of Cardiovascular Disease Later in Life, despite the Indisputable Presence of Multiple Cardiovascular Risk Factors at a Young Age. The Journal of Clinical Endocrinology \& Metabolism, 96, 3675. https://doi.org/10.1210/jc.2011-2935

[10] Robert, L. and Rosenfield, M.D. (2017) Etiology and Pathophysiology of Polycystic Ovary Syndrome in Adolescents.

https://www.uptodate.com/contents/etiology-and-pathophysiology-of-polycystic-ov ary-syndrome-in-adolescents

[11] Moran, L.J., Hutchison, S.K., Norman, R.J. and Teede, H.J. (2011) Lifestyle Changes in Women with Polycystic Ovary Syndrome. The Cochrane Database of Systematic Reviews, 2011, Article ID: CD007506. https://doi.org/10.1002/14651858.CD007506.pub3

[12] Pasquali, R., Antenucci, D., Casimirri, F., et al. (1989) Clinical and Hormonal Characteristics of Obese Amenorrheic Hyperandrogenic Women before and after Weight Loss. The Journal of Clinical Endocrinology \& Metabolism, 68, 173. https://doi.org/10.1210/jcem-68-1-173

[13] Huber-Buchholz, M.M., Carey, D.G. and Norman, R.J. (1999) Restoration of Reproductive Potential by Lifestyle Modification in Obese Polycystic Ovary Syndrome: Role of Insulin Sensitivity and Luteinizing Hormone. The Journal of Clinical Endocrinology \& Metabolism, 84, 1470.

[14] Barbieri, R.L. and Ehrmann, D.A. (2017) Treatment of Polycystic Ovary Syndrome in Adults.

https://www.uptodate.com/contents/treatment-of-polycystic-ovary-syndrome-in-ad ults 
[15] Dumesic, D.A. and Lobo, R.A. (2013) Cancer Risk and PCOS. Steroids, 78, 782. https://doi.org/10.1016/j.steroids.2013.04.004

[16] Nestler, J.E. (2008) Metformin for the Treatment of the Polycystic Ovary Syndrome. The New England Journal of Medicine, 358, 47-54. https://doi.org/10.1056/NEJMct0707092

[17] Morley, L.C., et al. (2017) Metformin Therapy for the Management of Infertility in Women with Polycystic Ovary Syndrome: Scientific Impact Paper No. 13. BJOG, 124, e306. https://doi.org/10.1111/1471-0528.14764

[18] Ferrannini, E. (2014) The Target of Metformin in Type 2 Diabetes. The New England Journal of Medicine, 371, 1547-1548. https://doi.org/10.1056/NEJMcibr1409796

[19] Madiraju, A.K., Erion, D.M., Rahimi, Y., et al. (2014) Metformin Suppresses Gluconeogenesis by Inhibiting Mitochondrial Glycerophosphate Dehydrogenase. Nature, 510, 542-546. https://doi.org/10.1038/nature13270

[20] Barbieri, R.L. and Ehrmann, D.A. (2017) Metformin for Treatment of the Polycystic Ovary Syndrome.

[21] Niafar, M., Hai, F., Porhomayon, J. and Nader, N.D. (2015) The Role of Metformin on Vitamin B12 Deficiency: A Meta-Analysis Review. Internal and Emergency Medicine, 10, 93-102. https://doi.org/10.1007/s11739-014-1157-5

[22] Kocak, M., Caliskan, E., Simsir, C. and Haberal, A. (2002) Metformin Therapy Improves Ovulatory Rates, Cervical Scores, and Pregnancy Rates in Clomiphene Citrate-Resistant Women with Polycystic Ovary Syndrome. Fertility and Sterility, 77, 101-106. https://doi.org/10.1016/S0015-0282(01)02941-7

[23] Vandermolen, D.T., Ratts, V.S., Evans, W.S., et al. (2001) Metformin Increases the Ovulatory Rate and Pregnancy Rate from Clomiphene Citrate in Patients with Polycystic Ovary Syndrome Who Are Resistant to Clomiphene Citrate Alone. Fertility and Sterility, 75, 310-315. https://doi.org/10.1016/S0015-0282(00)01675-7

[24] Moghetti, P., Castello, R., Negri, C., et al. (2000) Metformin Effects on Clinical Features, Endocrine and Metabolic Profiles, and Insulin Sensitivity in Polycystic Ovary Syndrome: A Randomized, Double-Blind, Placebo-Controlled 6-Month Trial, Followed by Open, Long-Term Clinical Evaluation. The Journal of Clinical Endocrinology \& Metabolism, 85, 139-146.

[25] Unlühizarci, K., Keleştimur, F., Bayram, F., et al. (1999) The Effects of Metformin on Insulin Resistance and Ovarian Steroidogenesis in Women with Polycystic Ovary Syndrome. Clinical Endocrinology, 51, 231-236. https://doi.org/10.1046/j.1365-2265.1999.00786.x

[26] Tang, T., Lord, J.M., Norman, R.J., et al. (2012) Insulin-Sensitising Drugs (Metformin, Rosiglitazone, Pioglitazone, D-Chiro-Inositol) for Women with Polycystic Ovary Syndrome, Oligo Amenorrhoea and Subfertility. The Cochrane Database of Systematic Reviews, No. 1, CD003053.

[27] Palomba, S., Orio, F.J., Falbo, A., Russo, T., Tolino, A. and Zullo, F. (2007) Clomiphene Citrate versus Metformin as First-Line Approach for the Treatment of Anovulation in Infertile Patients with Polycystic Ovary Syndrome. The Journal of Clinical Endocrinology \& Metabolism, 92, 3498-3503.

https://doi.org/10.1210/jc.2007-1009

[28] Morin-Papunen, L.C., Vauhkonen, I., Koivunen, R.M., et al. (2000) Endocrine and Metabolic Effects of Metformin versus Ethinyl Estradiol-Cyproterone Acetate in Obese Women with Polycystic Ovary Syndrome: A Randomized Study. The Journal of Clinical Endocrinology \& Metabolism, 85, 3161-3168. 
https://doi.org/10.1210/jc.85.9.3161

[29] Costello, M., Shrestha, B., Eden, J., et al. (2007) Insulin-Sensitising Drugs versus the Combined Oral Contraceptive Pill for Hirsutism, Acne and Risk of Diabetes, Cardiovascular Disease, and Endometrial Cancer in Polycystic Ovary Syndrome. The Cochrane Database of Systematic Reviews, No. 1, CD005552.

[30] Tso, L.O., Costello, M.F., Albuquerque, L.E., et al. (2009) Metformin Treatment before and during IVF or ICSI in Women with Polycystic Ovary Syndrome. The Cochrane Database of Systematic Reviews, 11, CD006105.

[31] Palomba, S., Falbo, A. and La Sala, G.B. (2013) Effects of Metformin in Women with Polycystic Ovary Syndrome Treated with Gonadotrophins for in Vitro Fertilisation and Intracytoplasmic Sperm Injection Cycles: A Systematic Review and Meta-Analysis of Randomised Controlled Trials. BJOG, 120, 267-276.

https://doi.org/10.1111/1471-0528.12070

[32] Palomba, S., Falbo, A., Carrillo, L., et al. (2011) Metformin Reduces Risk of Ovarian Hyperstimulation Syndrome in Patients with Polycystic Ovary Syndrome during Gonadotropin-Stimulated in Vitro Fertilization Cycles: A Randomized, Controlled Trial. Fertility and Sterility, 96, 1384-1390.

https://doi.org/10.1016/j.fertnstert.2011.09.020

[33] Al-Inany, H.G., Youssef, M.A., Ayeleke, R.O., et al. (2016) Gonadotrophin-Releasing Hormone Antagonists for Assisted Reproductive Technology. The Cochrane Database of Systematic Reviews, 4, CD001750. https://doi.org/10.1002/14651858.CD001750.pub4

[34] Practice Committee of the American Society for Reproductive Medicine, Practice Committee of the American Society for Reproductive Medicine (2017) Role of Metformin for Ovulation Induction in Infertile Patients with Polycystic Ovary Syndrome (PCOS): A Guideline. Fertility and Sterility, 108, 426-441. https://doi.org/10.1016/j.fertnstert.2017.06.026

[35] Lord, J.M., Flight, I.H. and Norman, R.J. (2003) Insulin-Sensitising Drugs (Metformin, Troglitazone, Rosiglitazone, Pioglitazone, D-Chiro-Inositol) for Polycystic Ovary Syndrome. The Cochrane Database of Systematic Reviews, No. 3, CD003053.

[36] Pasquali, R., Gambineri, A., Biscotti, D., et al. (2000) Effect of Long-Term Treatment with Metformin Added to Hypocaloric Diet on Body Composition, Fat Distribution, and Androgen and Insulin Levels in Abdominally Obese Women with and without the Polycystic Ovary Syndrome. The Journal of Clinical Endocrinology \& Metabolism, 85, 2767-2774. https://doi.org/10.1210/jcem.85.8.6738

[37] Kay, J.P., Alemzadeh, R., Langley, G., et al. (2001) Beneficial Effects of Metformin in Normoglycemic Morbidly Obese Adolescents. Metabolism, 50, 1457-1461. https://doi.org/10.1053/meta.2001.28078

[38] Freemark, M. and Bursey, D. (2001) The Effects of Metformin on Body Mass Index and Glucose Tolerance in Obese Adolescents with Fasting Hyperinsulinemia and a Family History of Type 2 Diabetes. Pediatrics, 107, e55.

https://doi.org/10.1542/peds.107.4.e55

[39] Harborne, L.R., Sattar, N., Norman, J.E. and Fleming, R. (2005) Metformin and Weight Loss in Obese Women with Polycystic Ovary Syndrome: Comparison of Doses. The Journal of Clinical Endocrinology \& Metabolism, 90, 4593-4598. https://doi.org/10.1210/jc.2004-2283

[40] Rowan, J.A., Hague, W.M., Gao, W., Battin, M.R. and Moore, M.P. (2008) Metformin versus Insulin for the Treatment of Gestational Diabetes. The New England Journal of Medicine, 358, 2003-2015. https://doi.org/10.1056/NEJMoa0707193 
[41] Morin-Papunen, L., Rantala, A.S., Unkila-Kallio, L., Tiitinen, A., Hippeläinen, M., Perheentupa, A., Tinkanen, H., Bloigu, R., Puukka, K., Ruokonen, A. and Tapanainen, J.S. (2012) Metformin Improves Pregnancy and Live-Birth Rates in Women with Polycystic Ovary Syndrome (PCOS): A Multicenter, Double-Blind, Placebo-Controlled Randomized Trial. The Journal of Clinical Endocrinology \& Metabolism, 97, 1492-1500. https://doi.org/10.1210/jc.2011-3061

\section{Abbreviation}

POCS: Polycystic Ovary Syndrome

NIH: National Institute of Health

HDL: High-Density Lipoproteins

LDL: Low-Density Lipoprotein

COCs: Combined Oral Hormonal Contraceptives 